\title{
Plastic modifications within inhibitory control networks induced by practicing a stop-signal task: An electrical neuroimaging study
}

\author{
Aurelie L. Manuel ${ }^{a}$, Fosco Bernasconi ${ }^{a}$ and Lucas Spierer ${ }^{a, b, *}$ \\ ${ }^{a}$ Neuropsychology and Neurorehabilitation Service, Vaudois University Hospital Center and University of Lausanne, Lausanne, Switzerland \\ ${ }^{\mathrm{b}}$ Neurology Unit, Department of Medicine, Faculty of Sciences, University of Fribourg, Fribourg, Switzerland
}

\begin{abstract}
Introduction: Inhibitory control refers to our ability to suppress ongoing motor, affective or cognitive processes and mostly depends on a fronto-basal brain network. Inhibitory control deficits participate in the emergence of several prominent psychiatric conditions, including attention deficit/hyperactivity disorder or addiction. The rehabilitation of these pathologies might therefore benefit from training-based behavioral interventions aiming at improving inhibitory control proficiency and normalizing the underlying neurophysiological mechanisms. The development of an efficient inhibitory control training regimen first requires determining the effects of practicing inhibition tasks.

Methods: We addressed this question by contrasting behavioral performance and electrical neuroimaging analyses of event-related potentials (ERPs) recorded from humans at the beginning versus the end of $1 \mathrm{~h}$ of practice on a stop-signal task (SST) involving the withholding of responses when a stop signal was presented during a speeded auditory discrimination task.

Results: Practicing a short SST improved behavioral performance. Electrophysiologically, ERPs differed topographically at $200 \mathrm{msec}$ post-stimulus onset, indicative of the engagement of distinct brain network with learning. Source estimations localized this effect within the inferior frontal gyrus, the pre-supplementary motor area and the basal ganglia. Conclusion: Our collective results indicate that behavioral and brain responses during an inhibitory control task are subject to fast plastic changes and provide evidence that highorder fronto-basal executive networks can be modified by practicing a SST.
\end{abstract}

\section{Introduction}

Inhibitory control refers to the ability to suppress ongoing cognitive, affective or motor processes (Dillon and Pizzagalli,
2007) and relies on a fronto-striato-basal network (Aron, 2011). Structural and functional deficits within the inhibitory control network have been repeatedly advanced as constituting a causal factor of, or at least as being associated with,

\footnotetext{
* Corresponding author. Neurology Unit, Department of Medicine, University of Fribourg, PER 09, Chemin du Musée 5, CH-1700 Fribourg, Switzerland.

E-mail address: lucas.spierer@gmail.com (L. Spierer).
} 
prominent psychiatric conditions, including for example attention deficit/hyperactivity (Overtoom et al., 2002), obsessive-compulsive disorders (Chamberlain et al., 2006) and addiction (Fillmore and Rush, 2002).

While inhibitory control has been extensively studied (Chambers et al., 2009; Aron, 2011), the behavioral and brain plastic changes induced by practicing inhibition tasks remain largely unresolved.

Manuel et al. (2010) demonstrated that training on a Go/ NoGo task improved inhibitory control performance, but that the behavioral improvement was not supported by a modification of the global fronto-basal inhibitory control network. Rather, neuroplastic changes manifested within temporoparietal cortices over the initial stages of the processing of the stimuli, indicative of the development of stimulus-driven, feed-forward forms of inhibition directly triggered by the NoGo stimuli (Manuel et al., 2010; Shiffrin and Schneider, 1977).

In the current study, we hypothesized that the global fronto-basal inhibitory control network could be modified by practicing inhibitory control with a stop-signal task (SST). SSTs consist in speeded discrimination tasks in which responses to the stimuli have to be canceled when a stop signal is presented (Logan and Cowan, 1984). By contrast to the Go/NoGo task used in Manuel et al. (2010), stimulus-response mappings are inconsistent in the SST (each Go stimulus is associated with activation or with inhibition goals). Therefore, automatic inhibition would unlikely develop during SST practice, and the fronto-basal inhibitory control network would be constantly involved (Verbruggen et al., 2008a) and in turn strengthened.

To test this hypothesis, we contrasted behavioral performance and electrical neuroimaging analyses of auditory event-related potentials (ERPs) to Go stimuli recorded at the beginning versus the end of a SST practice session.

\section{Materials and methods}

\subsection{Participants}

Thirteen right-handed volunteers participated in the study (7 male, mean age 23.9 years). No participant had a history of neurological or psychiatric illness and all reported normal hearing. Each participant provided written, informed consent to participate in the study. All procedures were approved by the local Ethics committee.

\subsection{Stimuli}

Auditory stimuli were $75 \mathrm{msec}$ band-pass noise bursts [410-470 Hz (Go 1), 592-652 Hz (Stop) and 850-910 Hz (Go 2); 5 msec rise/fall time; $44.1 \mathrm{kHz}$ sampling] presented via ER-4PEtymotic earphones.

\subsection{Procedure and task}

Participants completed an auditory SST (Logan and Cowan, 1984) in which they discriminated between the pitch of two stimuli (Go 1, low pitch, button 1; or Go 2, high pitch, button 2) as fast and accurately as possible via a manual response-box button, unless immediately followed by the stop-signal stimulus (Stop). Participants were seated in an electricallyshielded and sound-attenuated booth in front of a 19" liquid crystal display (LCD) screen. Stimulus delivery and response recording were controlled using E-prime 2.0. All trials began with an inter-trial interval (ITI) varying randomly between 2000 and $3000 \mathrm{msec}$, followed by the Go stimulus (either Go 1 or Go 2). During the ITI, a fixation cross was presented at the center of the screen. At the end of the ITI, the cross was turned off, the Go 1 or Go 2 sounds were presented and the time window during which responses were recorded was open. On $33 \%$ of the trials, a stop-signal tone (Stop) was presented shortly after the Go stimulus, which indicated that participants were to inhibit their response (see Fig. 1). These trials are referred to as "stop-signal trials" in contrast to the $66 \%$ of "Go trials" during which the response had to be executed to its end. On stop trials, the delay between the Go and the Stop stimulus (stop signal delay, SSD) was initially set at $300 \mathrm{msec}$ and adjusted continuously throughout each block with a tracking procedure allowing to obtain a probability of successfully stopping of .5 (Verbruggen and Logan, 2009). When participants managed to stop their response during a stop-signal trial, the SSD increased automatically by $50 \mathrm{msec}$; when they responded on a stop-signal trial, SSD decreased by $50 \mathrm{msec}$. The SSD was reset to 300 at the beginning of each block. The SST was divided in ten blocks each containing 102 randomly intermixed stop and go trials: 68 Go stimuli (34 Go 1, 34 Go 2) and 34 stop-signal trials. The whole stop-signal practice session included a total of 1020 stimuli and lasted for a total of about $1 \mathrm{~h}$.

\subsection{EEG acquisition and pre-processing}

Continuous electroencephalography (EEG) was acquired at $1024 \mathrm{~Hz}$ through a 128-channels Biosemi ActiveTwo system referenced to the common mode sense-driven right leg (CMSDRL) ground. EEG data pre-processing and analyses were conducted using Cartool software (http://sites.google.com/ site/fbmlab/cartool; Brunet et al., 2011). EEG epochs from $100 \mathrm{msec}$ pre- to $300 \mathrm{msec}$ post-stimulus onset were averaged, for each participant, for all Go stimuli and separately for the first four blocks (Beginning condition, BEG) and the four last blocks (End condition, END) of the SST task. This epoch of interest was chosen to reduce the contamination of the ERP to the Go stimuli by activity related to the stop signals. A $\pm 80 \mu \mathrm{V}$ automatic artifact rejection criterion was applied to exclude artifact epochs. Prior to group averaging, data at artifact electrodes from each participant were interpolated using 3D splines (mean 5.8\% interpolated electrodes; Perrin et al., 1987).

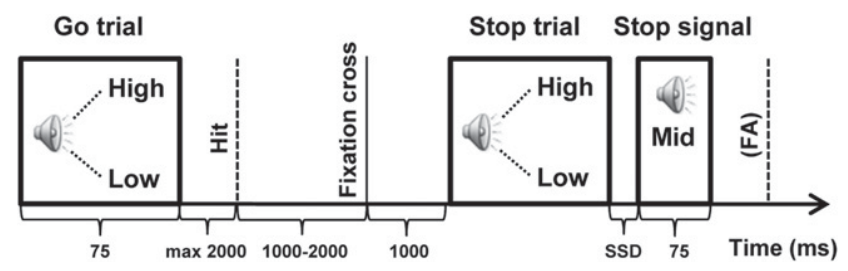

Fig. 1 - Experimental design. Each participant completed a 1-h practice session on the SST. The SSD varied according to participants' performance. 
Data were band-pass filtered $(.18-40 \mathrm{~Hz})$ and recalculated against the average reference. A baseline correction was then applied to the whole epoch. We did not sort trials as a function of performance or of whether it was followed by a stop signal or not because the type of trial (Go or stop trial) could not be predicted and our period of interest did not include the stop stimuli in stop trials. The average number ( \pm standard error of the mean (SEM)) of accepted epochs was $382 \pm 6$ for the Go trials at the BEG and $364 \pm 9$ for the Go trials in the END condition. These values did not statistically differ $(p>.05)$.

\subsection{Topographic patterns analyses}

A topographic pattern analysis was applied to the ERPs to determine whether the configuration of intracranial generators changed between the beginning and the end of the practice (e.g., Michel et al., 2004; Murray et al., 2008; Manuel et al., 2010, 2012). This approach is based on evidence that the ERP map topography does not vary randomly across time, but remains quasi-stable over $20-100 \mathrm{msec}$ functional microstates before rapidly switching to other stable periods (Lehmann and Skrandies, 1980; Britz and Michel, 2011). Because a change in the topography of the scalp-recorded electric field necessarily follows from a change in the configuration of the underlying brain's active generators, topographic modulations can be directly interpreted as the engagement of distinct brain networks (e.g., Lehmann, 1987). This method is independent of the reference electrode and is insensitive to pure amplitude modulations across conditions (topographies of normalized maps are compared; Tzovara et al., 2012 for discussion).

The sequence of predominating topographies (template maps) in the cumulative group-averaged data was identified using a hierarchical clustering based on an atomize and agglomerate approach. The optimal number of clusters to describe the dataset was identified using a modified Krzanowski-Lai criterion (Tibshirani et al., 2005). Differences in the pattern of topographic maps observed between conditions in the group-averaged data were tested by calculating the spatial correlation between these template maps from the group-averaged data and each time-point of single-subject data from each experimental condition. For this fitting procedure, each time-point of each ERP from each subject was labeled according to the map with which it best correlated spatially. The output of fitting is a measure of relative map presence in milliseconds, which indicates the amount of time over a given interval that each map, which was identified in the group-averaged data, best accounted for the response from a given individual subject and condition. These values were then submitted to a t-test between the BEG versus END condition (e.g., Murray et al., 2008 for details on the procedure).

\subsection{Electrical source estimations}

Electrical source estimations were calculated using a distributed linear inverse solution and the local autoregressive average (LAURA) regularization approach (Grave de Peralta et al., 2001, 2004). The calculation of the source estimations have been detailed elsewhere (e.g., Manuel et al., 2012; Thelen et al., 2012).
The results of the above topographic pattern analysis defined time periods over which intracranial sources were estimated and statistically processed. ERPs for each participant and condition were first averaged over the period of interest determined by the topographic pattern analysis. Then, intracranial sources were estimated for the resulting one time-sample for each participant and condition and then statistically compared at each solution point between the BEG versus END condition.

\section{Results}

\subsection{Behavioral results}

We indexed behavioral performance by the median Go RTs, SSDs and SSRTs (stop-signal reaction times; Fig.2). SSRT is calculated by subtracting the median SSD from the median response time (RT) (Band et al., 2003; Verbruggen and Logan, 2009). In line with previous literature, we considered the SSRT as the critical variable because it indexes the time needed to inhibit a response once the stop signal occurs, i.e., the latency of the stop process (Verbruggen and Logan, 2008b). As for the EEG analyses, behavioral data were separately averaged for the Beginning (BEG) and End (END) conditions. SSRT decreased significantly between the beginning and the end of the practice session [BEG: median \pm SEM = $177.5 \pm 7.2 \mathrm{msec}$; END: $\left.146.6 \pm 7.8 \mathrm{msec}^{\mathrm{t}} \mathrm{t}_{(12)}=2.72, p=.018\right]$. Go RTs, SSD, the percentage of successful stopping and of misses did not significantly differ with practice [Go RT: BEG: $756.3 \pm 28.6 \mathrm{msec}$, END: $778.3 \pm 30.9 \mathrm{msec}, \mathrm{t}_{(12)}=-.72, p=.483 ;$ SSD: BEG: $578.8 \pm 28.4 \mathrm{msec}$, END: $631.7 \pm 32.8 \mathrm{msec}$, $t_{(12)}=-1.85, p=.088$; percent success stop: BEG: $61.76 \pm 1.38 \%$, END: $63.34 \pm 1.67 \%, \mathrm{t}_{(12)}=-.90, p=.386$ and percent misses: BEG: $.30 \pm .09 \%$, END: $.63 \pm .17 \%, \mathrm{t}_{(12)}=-1.22, p=.243$ ].

\subsection{Electrical neuroimaging results}

\subsubsection{Topographic pattern analysis}

The output of the topographic pattern analysis is displayed in Fig. 3a [an exemplar ERP waveform ( $\mathrm{Cz}$ electrode) to the Go

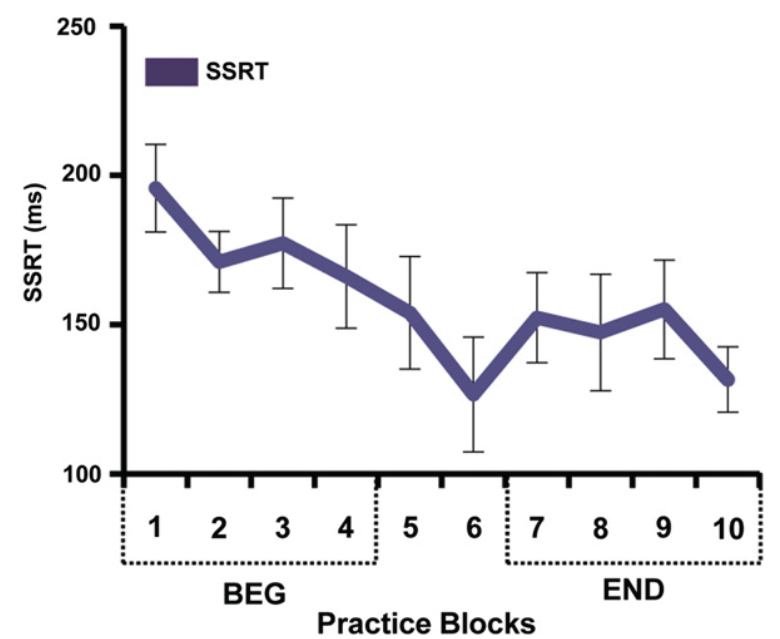

Fig. 2 - Behavioral results. The SSRT decreased with SST practice. 


\section{a Exemplar ERP waveform $(\mathrm{Cz})$ and temporal segmentation}

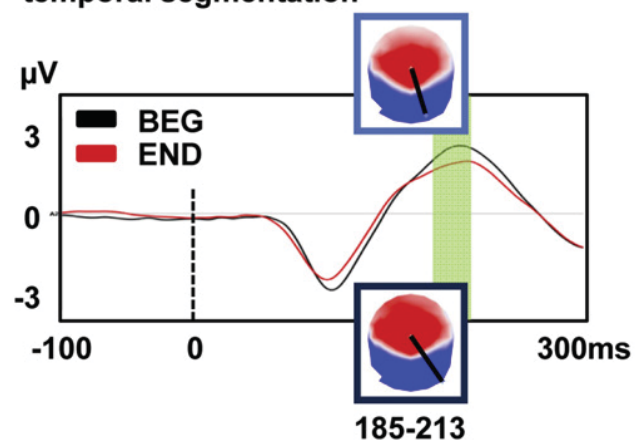

b Individual-subject fitting (185-213 ms)

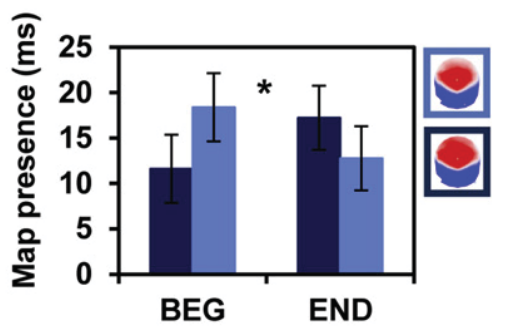

Fig. 3 - Topographic pattern analyses of the auditory evoked potential. a. The auditory ERPs in response to the beginning (BEG; black) and the end (END; red) of the SST training are displayed in microvolts as a function of time for an exemplar ERP waveform (Cz). The topographic pattern analysis identified one period of stable electric field topography where two different maps were observed for the BEG and the END conditions: 185-213 msec post-stimulus onset. b. The reliability of this observation at the group-averaged level was then assessed at the single-subject level using a spatial correlation fitting procedure. Over the 185-213 msec period, different maps (framed in light and dark blue, the black bars link the maxima with the minima of the topography) described the ERPs as a function of SST practice (BEG/END). Error bars indicate SEM.

stimuli is provided to help evaluating the signal quality and to situate the present effect relative to typical auditory ERP waveform components]. The global explained variance of the hierarchical clustering analysis was $97.8 \%$. This topographic pattern analysis identified the same sequence of stable topographic maps for group-averaged ERPs from the BEG and END conditions, except for the 185-213 msec poststimulus onset time period. Over this period, different maps were observed for the BEG versus END conditions. The reliability of this observation at the group-average level was then assessed at the single-subject level using a spatial correlation fitting procedure (see Method section). The individual-subject fitting procedure revealed that over the 185-213 msec period, the light blue map more frequently characterized the BEG and the dark blue map the END condition $\left[\mathrm{t}_{(12)}=-2.23, p<.05\right]$, indicative of the engagement of distinct configurations of intracranial generators in response to Go stimuli presented at the beginning versus the end of the SST practice (Fig. 3b).

\subsubsection{Electrical source estimations}

LAURA distributed source estimations revealed a significant decrease of activation between the BEG and END conditions within the right inferior frontal gyrus (rIFG) as well as in the pre-supplementary motor area (SMA), SMA, primary motor area (M1) and basal ganglia $\left[\mathrm{t}_{(12)}>3.05 ; p<.01\right.$; rIFG and left precentral local maxima at respectively $(44 ; 40 ; 10)$ and $(-23 ;-12 ; 77) \mathrm{mm}$ within the Montreal Neurological Institute (MNI) space; Fig. 4]. Although we applied statistically robust parametric mapping analyses of source estimations, our results mostly reveal modulations within subcortical areas, whose activity is possibly less reliably detected by scalprecorded EEG than superficial cortical activity. However, the source space used in the current study includes subcortical gray matter, and distributed source estimations calculate the current density at all solution points. Recent evidence demonstrates that deep sources can be reliably estimated from scalp-recorded electrophysiological data (Michel et al., 2004; Lucka et al., 2012).

\section{Discussion}

We showed that performance in a SST improves rapidly with practice and we identified spatio-temporal brain mechanisms of the supporting neuroplastic changes. Behaviorally, SSRT decreased over the course of the SST session, indicating a decrease in the speed of response inhibition. The contrast between electrical neuroimaging responses to Go stimuli recorded at the beginning versus the end of the SST session showed that the ERPs modulated topographically as a function of practice at a latency of $200 \mathrm{msec}$ post-stimulus onset, indicative of changes in the configuration of the underlying

Statistical analysis of LAURA Source estimations (BEG vs. END)

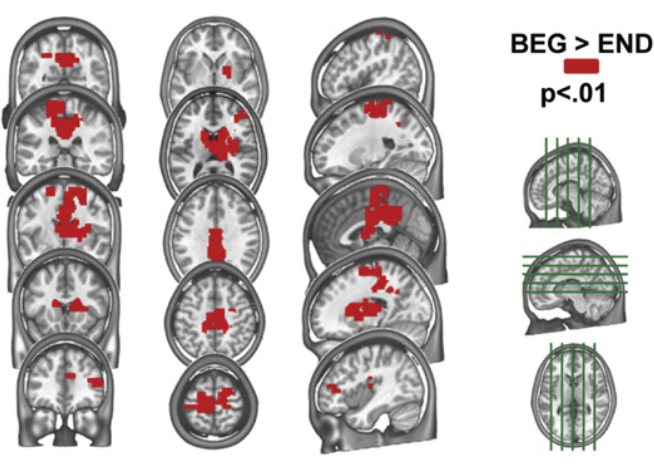

Fig. 4 - Electrical source analyses. a. Node-wise t-tests over the 185-213 msec post-stimulus onset period revealed significant decrease in activity between the beginning versus the end of SST session in the IIFG and the pre-SMA, SMA, and basal ganglia. 
intracranial generators. The statistical analysis of electrical source estimations showed that this effect followed from a decrease in the activity of the rIFG as well as of the pre-SMA, primary motor cortex and basal ganglia.

Our behavioral results corroborate previous psychophysical evidence for an improvement of SST performance with practice (Fillmore et al., 2001; Turner et al., 2004; though see Cohen and Poldrack, 2008). Because it takes into account both the SSD and Go RT, the SSRT is generally considered as being independent of changes in response strategies and thus to constitute a reliable index of SST proficiency (Congdon et al., 2012). However, SSRT has been shown to depend on several factors unrelated to inhibitory control including e.g., the probability or salience of stop trials (van den Wildenberg et al., 2002; van der Schoot et al., 2005) or the motivational context (Leotti and Wager, 2010), suggesting that it may not forcibly reflect inhibition performance. Although negative results should be interpreted with caution, the change in SSRT unlikely followed from a change in response strategy in the current study because there was no evidence for a change in the proportion of missed Go or of Stop success across training blocks.

At the electrophysiological level, the effects of practice manifested as a topographic modulation over the $185-213 \mathrm{msec}$ post-stimulus onset. The latency of our effect is in line with previous literature on the temporal dynamics of inhibitory control that reported that inhibition-related ERP components peak around $200 \mathrm{msec}$ post-stimulus in Go/NoGo (Falkenstein et al., 1999) and SST paradigms (Schmajuk et al., 2006).

Source estimations revealed that the topographic modulation followed from a change in the activity of the IFG and pre-SMA, primary motor cortex and basal ganglia. This result is highly consistent with previous functional, transcranial magnetic stimulation (TMS) and lesion studies showing a specific involvement of the rIFG, pre-SMA and basal ganglia in inhibitory control. Numerous studies indeed pointed out this right-lateralized fronto-basal network as the core network of inhibitory control of motor action (in SST task, see Aron and Poldrack, 2006; Verbruggen and Logan, 2008b; Aron, 2011). TMS over the rIFG (but not the left IFG or right middle frontal gyrus) has been shown to impair stopping performance (Chambers et al., 2006). Likewise, lesions in the rIFG or in the right pre-SMA lead to a decrease in stopping performance (Aron et al., 2003). Our finding for practiceinduced changes in the activity of the basal ganglia is in line with functional magnetic resonance imaging (fMRI) studies reporting activations within the subthalamic nucleus (STN) and the striatum during inhibitory control tasks (e.g., Aron and Poldrack, 2006). Similarly, lesions or deep-brain stimulation of the STN influences SSRT during SSTs (van den Wildenberg et al., 2006; Eagle et al., 2008).

The inhibitory control of motor actions across various effectors, and critically of non-motor cognitive and affective functions relies on the same fronto-basal network (and latency) as the network that was modified by practicing SST in the current study. Indeed, the $200 \mathrm{msec}$ time period has been shown to correspond to a processing stage when non-motor types of inhibition manifest, suggesting that effector- and function-independent inhibitory processes take place over this time window (e.g., Jackson et al., 2001). Moreover, the pre-
SMA, IFG and STN are also recruited for inhibiting or stopping eye movements (Chikazoe et al., 2007), speech (Xue et al., 2008) or other language-related processes (Xue et al., 2006). Growing evidence also reports the involvement of this fronto-basal network in the inhibitory control of thoughts, memory or emotion (Jonides et al., 1998; Depue et al., 2007; Dillon and Pizzagalli, 2007). Although speculative, since the same fronto-basal network as the one modified by SST practice in the current study is involved in the inhibition of other cognitive processes, the effects of SST practice would likely impact other inhibition-related functions. In line with this hypothesis, recent evidence showed that inhibitory control training with a motor task reduced risky behavior during subsequent gambling tasks (Verbruggen et al., 2012).

Most of previous studies showed that decreases in SSRT were associated with increases in the activity within right prefrontal areas (Aron and Poldrack, 2006; Rubia et al., 2007; though see Chao et al., 2009). By contrast, we show that improvement in SST performance was associated with a decrease in activity in the IFG and pre-SMA. A putative neurophysiological mechanism accounting for the direction of our effect is that practice yielded to the exclusion of irrelevant neural activity, in turn increasing the selectivity and thus the efficiency of the neural activity (Kelly and Garavan, 2005). In line with our results, decreases in frontal activity are typically reported following training on tasks involving high-order executive function as the one used in the current study (Beauchamp et al., 2003; Hempel et al., 2004), whereas increases in the activity within task-relevant brain regions were observed following training with lower-level motor or sensory tasks (Kelly et al., 2006). However, as mentioned above, we cannot rule out that changes in response strategies or in motivation during the SST practice impacted the SSRT and thus also account for the decrease in rIFG activity. Another limitation of the current study is that because the SSDs tended to decrease between the beginning and the end of the session, our contrast was possibly contaminated by a differential anticipation of the stop cues. Fronto-central N2 components manifesting over our period of topographic modulation are indeed sensitive to the formation of temporal expectations and to their violations (e.g., Rimmele et al., 2011; Wessel et al., 2012). While the resetting of the SSD to $300 \mathrm{msec}$ at the beginning of each block likely minimized the potential influence of temporal expectations, it possibly induced another confound: Since participants became more proficient with SST practice and that the SSD were reset to the same value at the beginning and at the end of the task, stopping their responses was likely easier at the end than at the beginning of the session.

Of note, practicing the SST in the current study modified higher-order, late-latency fronto-basal executive mechanisms. This pattern contrasts with previous evidence for the development of automatic, feed-forward forms of inhibition induced by training with a Go/NoGo task (Manuel et al., 2010). This difference in the effect of practice with an SST versus a Go/NoGo task likely follow from the fact that in SST task, Go stimuli are inconsistently associated with Go and NoGo goals, whereas in Go/NoGo task, repeated associations between NoGo stimuli and NoGo goals enable the development of stimulus-driven inhibition (Shiffrin and Schneider, 1977; 
Verbruggen and Logan, 2008a). The current study cannot disentangle the contribution of procedural learning in the observed effects. However, behavioral and brain changes induced by task familiarization have been reported to manifest at the very beginning of practice session, to have small effects, and to reach ceiling after less than 500 trials (e.g., Ortiz and Wright, 2010 or Segalowitz et al., 2001). Any contribution of procedural learning to the effects observed in the current study would thus most likely be minor. However, further studies are required to elucidate the precise dynamic, consolidation rate and persistence in time of the effects of SST practice.

\section{Author contributions}

LS and ALM designed and performed research, analyzed data, and wrote the paper. FB performed research and analyzed data.

\section{Acknowledgments}

This work was supported by a grant from the Swiss National Foundation for Science to LS (\#320030-143348). Cartool software (http://sites.google.com/site/fbmlab/cartool) has been programmed by Denis Brunet, from the Functional Brain Mapping Laboratory, Geneva, Switzerland, and supported by the Center for Biomedical Imaging (CIBM) of Geneva and Lausanne. Recordings were performed within the auspices of the Functional Electrical Neuroimaging Laboratory (www.unil. ch/fenl) directed by Prof. Micah Murray at the Vaudois University Hospital Center and University of Lausanne, Switzerland. We thank David A. Magezi and Micah M. Murray for their valuable comments on the manuscript.

\section{R E F E R E N C E S}

Aron AR. From reactive to proactive and selective control: Developing a richer model for stopping inappropriate responses. Biological Psychiatry, 69(12): e55-e68, 2011.

Aron AR and Poldrack RA. Cortical and subcortical contributions to Stop signal response inhibition: Role of the subthalamic nucleus. Journal of Neuroscience, 26(9): 2424-2433, 2006.

Aron AR, Fletcher PC, Bullmore ET, Sahakian BJ, and Robbins TW. Stop-signal inhibition disrupted by damage to right inferior frontal gyrus in humans. Nature Neuroscience, 6(2): 115-116, 2003.

Band GP, van der Molen MW, and Logan GD. Horse-race model simulations of the stop-signal procedure. Acta Psychologica, 112(2): 105-142, Feb 2003.

Beauchamp MH, Dagher A, Aston JA, and Doyon J. Dynamic functional changes associated with cognitive skill learning of an adapted version of the Tower of London task. NeuroImage, 20(3): 1649-1660, 2003.

Britz J and Michel CM. State-dependent visual processing. Frontiers in Psychology, 2: 370, 2011.

Brunet D, Murray MM, and Michel CM. Spatiotemporal analysis of multichannel EEG: CARTOOL. Computational Intelligence Neuroscience, 2011: 813-870, 2011.
Chamberlain SR, Fineberg NA, Blackwell AD, Robbins TW, and Sahakian BJ. Motor inhibition and cognitive flexibility in obsessive-compulsive disorder and trichotillomania. American Journal of Psychiatry, 163(7): 1282-1284, 2006.

Chambers CD, Bellgrove MA, Stokes MG, Henderson TR, Garavan H, Robertson IH, et al. Executive "brake failure" following deactivation of human frontal lobe. Journal of Cognitive Neuroscience, 18(3): 444-455, 2006.

Chambers CD, Garavan H, and Bellgrove MA. Insights into the neural basis of response inhibition from cognitive and clinical neuroscience. Neuroscience and Biobehavioral Reviews, 33(5): 631-646, 2009.

Chao HH, Luo X, Chang JL, and Li CS. Activation of the presupplementary motor area but not inferior prefrontal cortex in association with short stop signal reaction time-an intrasubject analysis. BMC Neuroscience, 10: 75, 2009.

Chikazoe J, Konishi S, Asari T, Jimura K, and Miyashita Y. Activation of right inferior frontal gyrus during response inhibition across response modalities. Journal of Cognitive Neuroscience, 19(1): 69-80, 2007.

Cohen JR and Poldrack RA. Automaticity in motor sequence learning does not impair response inhibition. Psychonomic Bulletin Review, 15(1): 108-115, 2008.

Congdon E, Mumford JA, Cohen JR, Galvan A, Canli T, and Poldrack RA. Measurement and reliability of response inhibition. Frontiers in Psychology, 3: 37, 2012.

Depue BE, Curran T, and Banich MT. Prefrontal regions orchestrate suppression of emotional memories via a twophase process. Science, 317(5835): 215-219, 2007.

Dillon DG and Pizzagalli DA. Inhibition of action, thought, and emotion: A selective neurobiological review. Applied and Preventive Psychology, 12(3): 99-114, 2007.

Eagle DM, Baunez C, Hutcheson DM, Lehmann O, Shah AP, and Robbins TW. Stop-signal reaction-time task performance: Role of prefrontal cortex and subthalamic nucleus. Cerebral Cortex, 18(1): 178-188, 2008.

Falkenstein M, Hoormann J, and Hohnsbein J. ERP components in Go/Nogo tasks and their relation to inhibition. Acta Psychologica, 101(2-3): 267-291, 1999.

Fillmore MT and Rush CR. Impaired inhibitory control of behavior in chronic cocaine users. Drug and Alcohol Dependence, 66(3): 265-273, 2002.

Fillmore MT, Rush CR, Kelly TH, and Hays L. Triazolam impairs inhibitory control of behavior in humans. Experimental and Clinical Psychopharmacology, 9(4): 363-371, 2001.

Grave de Peralta MR, Gonzalez AS, Lantz G, Michel CM, and Landis T. Noninvasive localization of electromagnetic epileptic activity. I. Method descriptions and simulations. Brain Topography, 14(2): 131-137, 2001.

Grave de Peralta MR, Murray MM, Michel CM, Martuzzi R, and Gonzalez Andino SL. Electrical neuroimaging based on biophysical constraints. NeuroImage, 21(2): 527-539, 2004.

Hempel A, Giesel FL, Garcia Caraballo NM, Amann M, Meyer H, Wustenberg $\mathrm{T}$, et al. Plasticity of cortical activation related to working memory during training. American Journal of Psychiatry, 161(4): 745-747, 2004.

Jackson GM, Swainson R, Cunnington R, and Jackson SR. ERP correlates of executive control during repeated language switching. Bilingualism Language Cognition, 4(2): 169-178, 2001.

Jonides J, Smith EE, Marshuetz C, Koeppe RA, and ReuterLorenz PA. Inhibition in verbal working memory revealed by brain activation. Proceedings of the National Academy of Sciences of the United States of America, 95(14): 8410-8413, 1998.

Kelly AM and Garavan H. Human functional neuroimaging of brain changes associated with practice. Cerebral Cortex, 15(8): 1089-1102, 2005.

Kelly C, Foxe JJ, and Garavan H. Patterns of normal human brain plasticity after practice and their implications for 
neurorehabilitation. Archives of Physical Medicine and Rehabilitation, 87(12): S20-S29, 2006.

Lehmann D. Principles of spatial analysis. In Gevins AS and Remond A (Eds), Handbook of Electroencephalography and Clinical Neurophysiology. Methods of Analysis of Brain Electrical and Magnetic Signals. Amsterdam: Elsevier, 1987: 309-354.

Lehmann D and Skrandies W. Reference-free identification of components of checkerboard-evoked multichannel potential fields. Electroencephalography and Clinical Neurophysiology, 48(6) 609-621, 1980.

Leotti LA and Wager TD. Motivational influences on response inhibition measures. Journal of Experimental Psychology Human Perception and Performance, 36(2): 430-447, 2010.

Logan GD and Cowan WB. On the ability to inhibit thought and action: A theory of an act of control. Psychological Review, 91(3): 295-327, 1984.

Lucka F, Pursiainen S, Burger M, and Wolters CH. Hierarchical Bayesian inference for the EEG inverse problem using realistic FE head models: Depth localization and source separation for focal primary currents. NeuroImage, 61(4): 1364-1382, 2012.

Manuel AL, Grivel J, Bernasconi F, Murray MM, and Spierer L. Brain dynamics underlying training-induced improvement in suppressing inappropriate action. Journal of Neuroscience, 30(41): 13670-13678, 2010.

Manuel AL, Bernasconi F, Murray MM, and Spierer L. Spatiotemporal brain dynamics mediating post-error behavioral adjustments. Journal of Cognitive Neuroscience, 24(6): 1331-1343, 2012.

Michel CM, Murray MM, Lantz G, Gonzalez S, Spinelli L, and Grave de PR. EEG source imaging. Clinical Neurophysiology, 115(10): 2195-2222, 2004

Murray MM, Brunet D, and Michel CM. Topographic ERP analyses: A step-by-step tutorial review. Brain Topography, 20(4): 249-264, 2008.

Ortiz JA and Wright BA. Differential rates of consolidation of conceptual and stimulus learning following training on an auditory skill. Experimental Brain Research., 201(3): 441-451, 2010 Mar

Overtoom CC, Kenemans JL, Verbaten MN, Kemner C, van der Molen MW, van EH, et al. Inhibition in children with attentiondeficit/hyperactivity disorder: A psychophysiological study of the stop task. Biological Psychiatry, 51(8): 668-676, 2002.

Perrin F, Pernier J, Bertrand O, Giard MH, and Echallier JF. Mapping of scalp potentials by surface spline interpolation. Electroencephalography and Clinical Neurophysiology, 66(1): 75-81, 1987.

Rimmele J, Jolsvai H, and Sussman E. Auditory target detection is affected by implicit temporal and spatial expectations. Journal of Cognitive Neuroscience, 23(5): 1136-1147, 2011.

Rubia K, Smith AB, Taylor E, and Brammer M. Linear agecorrelated functional development of right inferior frontostriato-cerebellar networks during response inhibition and anterior cingulate during error-related processes. Human Brain Mapping, 28(11): 1163-1177, 2007

Schmajuk M, Liotti M, Busse L, and Woldorff MG. Electrophysiological activity underlying inhibitory control processes in normal adults. Neuropsychologia, 44(3): 384-395, 2006.

Segalowitz SJ, Wintink AJ, and Cudmore LJ. P3 topographical change with task familiarization and task complexity. Brain Research Cognitive Brain Research, 12(3): 451-457, 2001.

Shiffrin RM and Schneider W. Controlled and automatic human information processing. II. Perceptual learning, automatic attending, and a general theory. Psychological Review, 84(2): 127-190, 1977.

Thelen A, Cappe C, and Murray MM. Electrical neuroimaging of memory discrimination based on single-trial multisensory learning. NeuroImage, 62(3): 1478-1488, 2012.

Tibshirani R, Walther G, Botstein D, and Brown P. Cluster validation by prediction strength. Journal of Computational Graphical Statistics, 14: 511-528, 2005.

Turner DC, Clark L, Dowson J, Robbins TW, and Sahakian BJ. Modafinil improves cognition and response inhibition in adult attention-deficit/hyperactivity disorder. Biological Psychiatry, 55(10): 1031-1040, 2004.

Tzovara A, Murray MM, Michel CM, and De Lucia M. A tutorial review of electrical neuroimaging from group-average to single-trial event-related potentials. Developmental Neuropsychology, 37(6): 518-544, 2012.

van den Wildenberg WP, van der Molen MW, and Logan GD. Reduced response readiness delays stop signal inhibition. Acta Psychologica, 111(2): 155-169, 2002.

van den Wildenberg WP, van Boxtel GJ, van der Molen MW, Bosch DA, Speelman JD, and Brunia CH. Stimulation of the subthalamic region facilitates the selection and inhibition of motor responses in Parkinson's disease. Journal of Cognitive Neuroscience, 18(4): 626-636, 2006.

van der Schoot M, Licht R, Horsley TM, and Sergeant JA. Effects of stop signal modality, stop signal intensity and tracking method on inhibitory performance as determined by use of the stop signal paradigm. Scandinavian Journal of Psychology, 46(4): 331-341, 2005.

Verbruggen F and Logan GD. Automatic and controlled response inhibition: Associative learning in the go/no-go and stopsignal paradigms. Journal of Experimental Psychology General, 137(4): 649-672, 2008a.

Verbruggen $\mathrm{F}$ and Logan GD. Response inhibition in the stop-signal paradigm. Trends in Cognitive Science, 12(12): 418-424, 2008b.

Verbruggen F and Logan GD. Models of response inhibition in the stop-signal and stop-change paradigms. Neuroscience and Biobehavioral Reviews, 33(5): 647-661, 2009.

Verbruggen F, Adams R, and Chambers CD. Proactive motor control reduces monetary risk taking in gambling. Psychological Science, 23(7): 805-815, 2012.

Wessel JR, Danielmeier C, Morton JB, and Ullsperger M. Surprise and error: Common neuronal architecture for the processing of errors and novelty. Journal of Neuroscience, 32(22): 7528-7537, 2012.

Xue G, Poldrack RA, and Aron AR. Right inferior frontal cortex is involved in inhibiting both manual and vocal responses. Society for Neuroscience Abstracts, 32: 367.5, 2006.

Xue G, Aron AR, and Poldrack RA. Common neural substrates for inhibition of spoken and manual responses. Cerebral Cortex, 18(8): 1923-1932, 2008. 